Zu Recht bezweifelt der Autor, ob die Schaffung einer internationalen Strafjustiz, wie sie seit den Nürnberger und Tokioter Prozessen diskutiert wird, das Problem der impunidad beseitigen kann, wird im allgemeinen doch nur die individuelle strafrechtliche Verantwortlichkeit der Täter vor einem Internationalen Strafgerichtshof in Erwägung gezogen, ohne zusätzlich den individuellen Rechtsschutz der Opfer zu berücksichtigen. Dieser soll wiederum nur über einzelne Staaten oder über Staatengemeinschaften, z.B. die UNO, ermöglicht werden, einschließlich aller darin liegender möglicher diplomatischer Implikationen.

Andererseits, und dieser Aspekt sollte bei der Diskussion nicht unterschlagen werden, allein schon die Tatsache, daß sich lateinamerikanische Regierungen veranlaßt sehen, überhaupt nunmehr derartige impunidad-Vorschriften zu erlassen, zeigt, daß sie unter erheblichen Rechtfertigungsdruck geraten sind, sowohl auf nationaler wie auf internationaler Ebene. Und dieses Buch kann dazu beitragen, daß die Straflosigkeit schwerer Menschenrechtsverletzungen nicht einfach mehr so dekretiert werden kann. Mittlerweile, und das verdient besonders hervorgerufen zu werden, ist eine Übersetzung dieser Untersuchung in spanischer Sprache erschienen, welcher man besonders in Lateinamerika, aber nicht nur dort, eine weite Verbreitung wünschen möchte.

Jürgen Saligmann

\title{
Juha Räikkä
}

\section{Do We Need Minority Rights?}

Conceptual Issues

Martinus Nijhoff Publishers, The Hague / Boston / London, 1996, 240 S., $£$ 69,00

(International Studies in Human Rights, Vol. 46)

Unter dem provokanten Titel "Do we need minority rights?" beschäftigt sich der Sammelband in acht Beiträgen mit dem Thema des Minderheitenschutzes. Vier der Autoren untersuchen das Thema unter philosophischen Aspekten, die weiteren Aufsätze präsentieren konkrete juristische, politische und praxisorientierte Probleme des Minderheitenschutzes.

Gibt es eine moralische Verpflichtung, Minderheiten zu schützen? Welche Minderheiten bedürfen des Schutzes, und nach welchen Kriterien sollen sie ausgewählt werden? Müssen Rechtstheorien die Rechte von Gruppen berücksichtigen und in welcher Form? Herausgeber Juha Räikka beschäftigt sich in seinem Beitrag mit der seit den 70er Jahren von Philosophen geführten Diskussion, ob eine Theorie der Gerechtigkeit nur dann als legitim anzusehen sei, wenn sie Minderheiten und Gruppen ausdrücklich berücksichtige. Der Autor kritisiert die pauschale Ablehnung jener Theorien der Gerechtigkeit, die Minderheiten nicht berücksichtigen, ohne zu hinterfragen, worauf eine Verpflichtung zum Schutz von Minderheiten basieren könnte. 
Wesley E. Cooper setzt sich in seinem Beitrag mit den Theorien von John Rawls und Will Kymlika auseinander. Er spricht sich gegen einen längerfristigen Minderheitenschutz aus, da dieser 'symbolische' Schranken zwischen den einzelnen Gruppen in einem Staat aufbauen könnte. Seiner Ansicht nach muß die Verwirklichung von gleichen Rechten für alle Bürger im Vordergrund stehen, nur kurzfristig seien zur Erreichung dieses Zieles unterstützende Maßnahmen für Minderheiten sinnvoll. Auch Marlies Galenkamp plädiert dafür, Benachteiligungen von Gruppen eher auf einer temporären als auf einer permanenten Basis auszugleichen, um die soziale Gerechtigkeit innerhalb eines Staates nicht aus dem Gleichgewicht zu bringen. Ein Minderheitenschutz sollte sich nicht aus den 'Wünschen' der verschiedenen Gruppen ergeben, sondern ausschlaggebend sollte die 'Notwendigkeit' eines solchen Schutzes sein.

Andres Follesdal versucht die Theorie des liberalen Kontraktualismus in Einklang zu bringen mit einigen zentralen Aspekten der Minderheitenproblematik. Seiner Ansicht nach sind Minderheitenrechte legitim, solange sie nötig und geeignet sind, wichtige Interessen zu schützen. Jedoch sollte Gruppen, die sich nicht den liberalen Werten von Gleichheit und Freiheit verpflichtet fühlen, keine speziellen Rechte gewährt werden.

Melissa S. Williams befürwortet in ihrem Beitrag eine verstärkte Repräsentation von Minderheiten in politischen Organen. Sie widerspricht der Befürchtung, mit Forderungen nach größerer politischer Partizipation Tür und Tor zu einer Fragmentierung der Gesellschaft und einer Überbetonung der Gruppeninteressen vor dem Gemeinwohl zu öffnen. Nach ihrer Definition zählen zu den politisch unterrepräsentierten Gruppen diejenigen, deren Mitglieder bereits in der Vergangenheit diskriminiert wurden und auch in der Gegenwart im Vergleich zu anderen Gruppen unter Benachteiligungen zu leiden haben.

John Packers Aufsatz "On the content of minority rights" unternimmt den Versuch, den Inhalt der Minderheitenrechte in den gegenwärtigen internationalen Beziehungen zu verdeutlichen. Er beginnt mit dem vieldiskutierten Problem, eine Minderheit zu definieren und spricht sich dafür aus, Minderheiten nicht nach objektiven Kriterien wie z.B. Sprache zu unterscheiden, sondern nach subjektiven Kriterien ${ }^{1}$. Im Lichte der Menschenrechtsphilosophie vergleicht er die Ansätze der kontraktualistischen und der kommunitaristischen Theorien hinsichtlich des Minderheitenschutzes und untersucht sodann ihre tatsächliche Verwirklichung im positiven Recht.

Peter Swan setzt sich in seinem Aufsatz mit der Rolle von Minderheiten und ökologischer Gerechtigkeit auseinander. Er fordert ein gruppenspezifisches Umweltrecht, da Minderheiten häufig stärker als andere Teile der Gesellschaft von Umweltbelastungen betroffen sind. In Erkenntnis dessen geht er der Frage nach, ob die ökologische Situation von Minderheiten dadurch zu verbessern sei, daß ihnen ein Mitspracherecht in ökologischen Entscheidungsprozessen eingeräumt werde.

1

"... 'the' or 'a minority' is a group of people who freely associate for an established purpose where their shared desire differs from that expressed by the majority rule." (S. 123). 
John F. Corvino gibt in seinem Aufsatz eine Anleitung, wie man nicht für die Rechte der homosexuellen Minderheit argumentieren sollte. Ausgehend von einem Überblick zu aktuellen Studien, die die Entstehung von Homosexualität untersuchen, argumentiert Corvino, daß die Wurzeln der Homosexualität größtenteils irrelevant für die moralische und rechtliche Diskussion seien. Er empfindet es als riskant, die Argumente auf eine mögliche genetische oder biologische Basis der Homosexualität zu stützen, da zum einen die wissenschaftlichen Studien durch neue Forschungen an Wert verlieren könnten, zum anderen der Glaube, es handele sich um eine angeborene "Krankheit", damit genährt würden. Daher empfiehlt er, Analogien zu den Argumenten der religiösen Minderheiten zu ziehen, da seiner Meinung nach sowohl Religion als auch Homosexualität auf tiefen Überzeugungen beruhen, auf einer Wahl basieren und oft versteckt ausgeübt werden müssen.

Der Sammelband befaßt sich mit einem aktuellen und wichtigen Thema, das zahlreiche, noch ungelöste Fragen aufwirft. Die vielfältige und kontroverse Herangehensweise der Autoren eröffnet neue Blickwinkel und stellt einen guten Beitrag zu der gegenwärtigen Diskussion zur Problematik der Minderheitenrechte dar. Als Einführungslektüre für den an diesem Thema interessierten Leser bietet sich das Werk jedoch nicht an, da die meisten Aufsätze eingehende Kenntnisse der philosophischen Debatte voraussetzen.

Birgit Schröder

\section{Edward Lawson}

Encyclopedia of Human Rights (2. ed.)

Taylor \& Francis, Washington, D.C. and London, 1996, 1715 pp., $£ 239,00$

Die Wahrung der Menschenrechte spielt in der heutigen Zeit in der internationalen Politik eine größere Rolle als jemals zuvor. Obwohl die Universal Declaration of Human Rights schon 1948 von der Generalversammlung der Vereinten Nationen verabschiedet wurde, war es um die tatsächliche Situation der Menschenrechte in weiten Teilen der Welt schlecht bestellt. Auch heute werden die Menschenrechte noch nicht überall geachtet, aber ihr Gewicht in den internationalen Beziehungen ist gestiegen. Zudem wird der Begriff der Menschenrechte weiter gefaßt. Es zählen nicht nur die Grundrechte wie u.a. Meinungs- und Versammlungsfreiheit, das Verbot rassischer Diskriminierung, das Verbot der Folter und die Gleichberechtigung der Geschlechter dazu, sondern zunehmend auch soziale Rechte, wie z.B. das Recht auf Entwicklung und soziale Gerechtigkeit (vgl. Declaration on Social Progress and Development).

Nach dem Ende des Ost-West-Konfliktes wurden die Menschenrechte zu einem wichtigen Bestandteil der Politik der westlichen Geberstaaten gegenüber den Entwicklungsländern. Erstmals wurde unter dem Schlagwort "Politische Konditionalität" die Wahrung der fun- 